Acta Crystallographica Section E

Structure Reports

Online

ISSN 1600-5368

\section{3-(Benzimidazolium-2-yl)propionate dihydrate}

\section{Xin Xiao, ${ }^{a}$ Yun-Qiang Zhang, ${ }^{a}$ Sai-Feng Xue ${ }^{a}$ and Zhu $\mathrm{Tao}^{\mathrm{b} *}$}

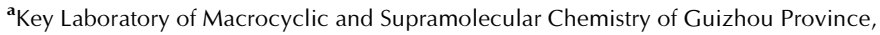
Guizhou University, Guiyang 550025, People's Republic of China, and ' Institute of Applied Chemistry, Guizhou University, Guiyang 550025, People's Republic of China

Correspondence e-mail: gyhxxiaoxin@163.com

Received 4 September 2008; accepted 27 September 2008

Key indicators: single-crystal X-ray study; $T=273 \mathrm{~K}$; mean $\sigma(\mathrm{C}-\mathrm{C})=0.003 \AA$; $R$ factor $=0.047 ; w R$ factor $=0.113 ;$ data-to-parameter ratio $=12.2$.

In the crystal struture of the title compound, $\mathrm{C}_{10} \mathrm{H}_{10} \mathrm{~N}_{2} \mathrm{O}_{2} \cdot 2 \mathrm{H}_{2} \mathrm{O}$, the component species are linked to the water molecules by $\mathrm{N}-\mathrm{H} \cdots \mathrm{O}$ and $\mathrm{O}-\mathrm{H} \cdots \mathrm{O}$ hydrogen bonds to form a three-dimensional network structure.

\section{Related literature}

For general background, see: Day \& Arnold (2000); Day et al. (2002); Freeman et al. (1981); Kim et al. (2000). For related structure, see: Ge et al. (2007).<smiles>O=C([O-])CCc1[nH]c2ccccc2[nH+]1</smiles>

\section{Experimental}

\section{Crystal data}

$\mathrm{C}_{10} \mathrm{H}_{10} \mathrm{~N}_{2} \mathrm{O}_{2} \cdot 2 \mathrm{H}_{2} \mathrm{O}$

$M_{r}=226.23$

Monoclinic, $P 2_{1} / c$

$a=18.444(3) \AA$

$b=4.9730(8) \AA$

$c=11.9097(19) \AA$

$\beta=94.530(5)^{\circ}$

\section{Data collection}

Bruker SMART APEXII CCD area-detector diffractometer Absorption correction: none 7787 measured reflections
2002 independent reflections 1394 reflections with $I>2 \sigma(I)$ $R_{\text {int }}=0.066$
$Z=4$

Mo $K \alpha$ radiation

$0.29 \times 0.26 \times 0.20 \mathrm{~mm}$
$T=273(2) \mathrm{K}$
Refinement

$R\left[F^{2}>2 \sigma\left(F^{2}\right)\right]=0.047$

$w R\left(F^{2}\right)=0.113$

$S=1.08$

1971 reflections

161 parameters

6 restraints

$\mathrm{H}$ atoms treated by a mixture of independent and constrained refinement

$\Delta \rho_{\max }=0.19 \mathrm{e} \AA^{-3}$

$\Delta \rho_{\min }=-0.23 \mathrm{e}^{-3}$

Table 1

Hydrogen-bond geometry $\left(\AA{ }^{\circ}\right)$.

\begin{tabular}{lllll}
\hline$D-\mathrm{H} \cdots A$ & $D-\mathrm{H}$ & $\mathrm{H} \cdots A$ & $D \cdots A$ & $D-\mathrm{H} \cdots A$ \\
\hline $\mathrm{N} 1-\mathrm{H} 1 \mathrm{~N} \cdots \mathrm{O} 1^{\mathrm{i}}$ & 0.86 & 1.86 & $2.700(2)$ & 166 \\
$\mathrm{~N} 2-\mathrm{H} 2 \mathrm{~N} \cdots \mathrm{O} 2^{\mathrm{ii}}$ & 0.86 & 1.80 & $2.654(3)$ & 170 \\
$\mathrm{O} 2 W-\mathrm{H} 2 W 1 \cdots \mathrm{O} 1 W^{\text {iii }}$ & $0.841(17)$ & $2.007(18)$ & $2.842(3)$ & $172(3)$ \\
\hline Symmetry codes: (i) $x, y+1, z$; (ii) $x,-y+\frac{1}{2}, z+\frac{1}{2} ;$ (iii) $-x+1, y-\frac{1}{2},-z+\frac{1}{2}$.
\end{tabular}

Data collection: APEX2 (Bruker, 2004); cell refinement: SAINT (Bruker, 2004); data reduction: $S A I N T$; $\operatorname{program}(\mathrm{s})$ used to solve structure: SHELXS97 (Sheldrick, 2008); program(s) used to refine structure: SHELXL97 (Sheldrick, 2008); molecular graphics: ORTEP-3 for Windows (Farrugia, 1997); software used to prepare material for publication: WinGX (Farrugia, 1999).

The authors gratefully acknowledge the Natural Science Foundation of China (grant No. 20767001), the International Collaborative Project of Guizhou Province, the Governor Foundation of Guizhou Province and the Natural Science Youth Foundation of Guizhou University (grant No. 2007-005) for financial support.

Supplementary data and figures for this paper are available from the IUCr electronic archives (Reference: NG2490).

\title{
References
}

Bruker (2004). APEX2 and SAINT. Bruker AXS Inc., Madison, Wisconsin, USA.

Day, A. I. \& Arnold, A. P. (2000). Int. Patent WO/2000/068232.

Day, A. I., Blanch, R. J., Arnold, A. P., Lorenzo, S., Lewis, G. R. \& Dance, I. (2002). Angew. Chem. Int. Ed. 41, 275-277.

Farrugia, L. J. (1997). J. Appl. Cryst. 30, 565.

Farrugia, L. J. (1999). J. Appl. Cryst. 32, 837-838.

Freeman, W. A., Mock, W. L. \& Shih, N. Y. (1981). J. Am. Chem. Soc. 103, $7367-7368$.

Ge, J. Y., Xue, S. F., Zhu, Q. J., Tao, Z. \& Zhang, J., X. (2007). J. Incl. Phenom. Macro. 58, 63-69.

Kim, J., Jung, I.-S., Kim, S.-Y., Lee, E., Kang, J.-K., Sakamoto, S., Yamaguchi, K. \& Kim, K. (2000). J. Am. Chem. Soc. 122, 540-541.

Sheldrick, G. M. (2008). Acta Cryst. A64, 112-122.

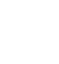




\section{supporting information}

Acta Cryst. (2008). E64, o2066 [doi:10.1107/S1600536808031346]

\section{3-(Benzimidazolium-2-yl)propionate dihydrate}

\section{Xin Xiao, Yun-Qiang Zhang, Sai-Feng Xue and Zhu Tao}

\section{S1. Comment}

As part of our ongoing investigation on benzimidazole compounds, we present a compound containing multiple functional groups that can develop strong intermolecular interactions with cucurbit $[n]$ urils $(\mathrm{CB}[n])$ (Freeman et al., 1981; Day \& Arnold, 2000; Day et al., 2002; Kim et al., 2000; Ge et al., 2007).

The crystal structure of the title compound (Fig. 1) consists of a 3-(1H-benzo[ $d]$ imidazol-2-yl) propanoic acid organic molecule and two lattice water molecules. the dihedral angle between the benzene ring $(\mathrm{C} 1, \mathrm{C} 2, \mathrm{C} 3, \mathrm{C} 4, \mathrm{C} 5, \mathrm{C} 6)$ and the imidazole ring $(\mathrm{C} 5, \mathrm{C} 6, \mathrm{C} 9, \mathrm{~N} 2, \mathrm{C} 7, \mathrm{~N} 1)$ is $0.61(13)^{\circ}$. The $\mathrm{C} 7-\mathrm{C} 8-\mathrm{C} 9-\mathrm{C} 10$ torsion angle is $-66.3(3)^{\circ}$. The title compound forms intermolecular $\mathrm{H}$ bonds whereas the protonated $\mathrm{N} 1$ and $\mathrm{N} 2$ atoms act as hydrogen-bond donors and the $\mathrm{O} 1$ and $\mathrm{O} 2$ atoms act as hydrogen-bond acceptors, the $\mathrm{O}-\mathrm{H} \cdots \mathrm{O}$ hydrogen bonds are also observed between the water molecules $\mathrm{O} 2 \mathrm{~W}$ and $\mathrm{O} 1 \mathrm{~W}$ (Table 1). these contacts and the cross-linking interactions stabilize the crystal packing.

\section{S2. Experimental}

The propionic anhydride (13 g, $0.1 \mathrm{~mol})$ was dissolved in hot water $(100 \mathrm{ml})$ with stirring, and a warm solution of 1,2-diaminobenzene( $10.8 \mathrm{~g}, 0.1 \mathrm{~mol})$ in 1,4-dioxane $(100 \mathrm{ml})$ was added, following by the addition of ployphosphoric acid (50 $\mathrm{ml})$ as catalyst. The mixture was refluxed for $8 \mathrm{~h}$ and then cooled, the solution was filtered and the filtrate was set aside for three weeks to obtain colorless crystals.

\section{S3. Refinement}

Water $\mathrm{H}$ atoms were located in a difference Fourier map and refined as riding in their as-found positions relative to $\mathrm{O}$ atoms with $U_{\text {iso }}(\mathrm{H})=1.2 U_{\text {eq }}(\mathrm{O})$. All other $\mathrm{H}$ atoms were placed in calculated positions and refined as riding, with $\mathrm{C}-\mathrm{H}=$ $0.93-0.97 \AA, \mathrm{N}-\mathrm{H}=0.86 \AA$, and $U_{\text {iso }}(\mathrm{H})=1.2-1.5 U_{\text {eq }}(\mathrm{C}, N)$. 


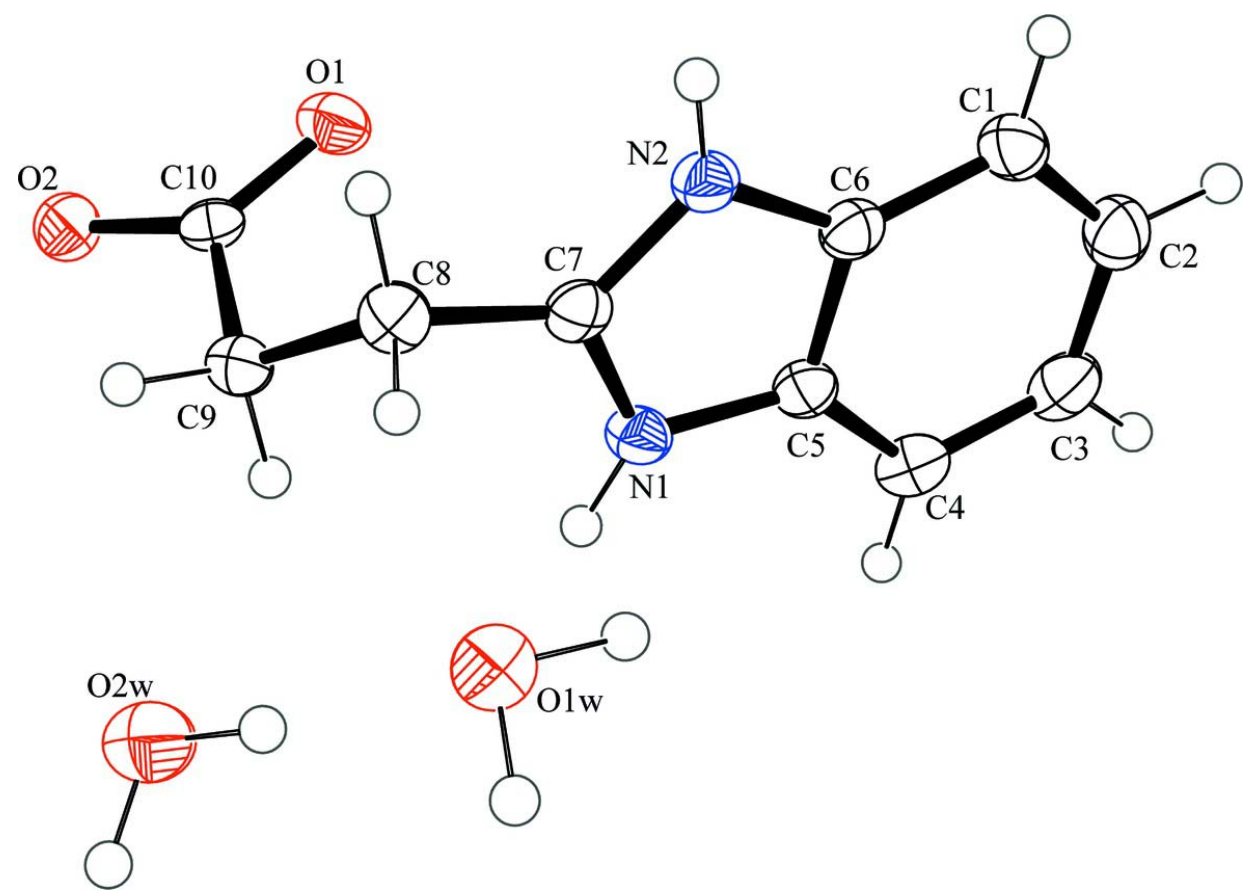

\section{Figure 1}

The molecular structure of (I) showing the atom-labelling scheme. Displacement ellipsoids are drawn at the 50\% probability level.

\section{3-(Benzimidazolium-2-yl)propionate dihydrate}

Crystal data

$\mathrm{C}_{10} \mathrm{H}_{10} \mathrm{~N}_{2} \mathrm{O}_{2} \cdot 2 \mathrm{H}_{2} \mathrm{O}$

$M_{r}=226.23$

Monoclinic, $P 2_{1} / c$

Hall symbol: -P $2 \mathrm{ybc}$

$a=18.444$ (3) $\AA$

$b=4.9730(8) \AA$

$c=11.9097$ (19) $\AA$

$\beta=94.530(5)^{\circ}$

$V=1089.0(3) \AA^{3}$

$Z=4$

\section{Data collection}

Bruker CCD area-detector diffractometer

Radiation source: fine-focus sealed tube Graphite monochromator $\varphi$ and $\omega$ scans

7787 measured reflections

2002 independent reflections
$F(000)=480$

$D_{\mathrm{x}}=1.380 \mathrm{Mg} \mathrm{m}^{-3}$

Mo $K \alpha$ radiation, $\lambda=0.71073 \AA$

Cell parameters from 2002 reflections

$\theta=1.1-25.4^{\circ}$

$\mu=0.11 \mathrm{~mm}^{-1}$

$T=273 \mathrm{~K}$

Prism, colourless

$0.29 \times 0.26 \times 0.20 \mathrm{~mm}$

1394 reflections with $I>2 \sigma(I)$

$R_{\text {int }}=0.066$

$\theta_{\text {max }}=25.4^{\circ}, \theta_{\text {min }}=1.1^{\circ}$

$h=-21 \rightarrow 19$

$k=-5 \rightarrow 4$

$l=-13 \rightarrow 14$ 


\section{Refinement}

Refinement on $F^{2}$

Least-squares matrix: full

$R\left[F^{2}>2 \sigma\left(F^{2}\right)\right]=0.047$

$w R\left(F^{2}\right)=0.113$

$S=1.08$

1971 reflections

161 parameters

6 restraints

Primary atom site location: structure-invariant direct methods
Secondary atom site location: difference Fourier map

Hydrogen site location: inferred from neighbouring sites

$\mathrm{H}$ atoms treated by a mixture of independent and constrained refinement

$w=1 /\left[\sigma^{2}\left(F_{\mathrm{o}}^{2}\right)+(0.0354 P)^{2}+0.5171 P\right]$ where $P=\left(F_{\mathrm{o}}{ }^{2}+2 F_{\mathrm{c}}{ }^{2}\right) / 3$

$(\Delta / \sigma)_{\max }<0.001$

$\Delta \rho_{\max }=0.19 \mathrm{e} \AA^{-3}$

$\Delta \rho_{\min }=-0.23 \mathrm{e} \AA^{-3}$

\section{Special details}

Geometry. All e.s.d.'s (except the e.s.d. in the dihedral angle between two 1.s. planes) are estimated using the full covariance matrix. The cell e.s.d.'s are taken into account individually in the estimation of e.s.d.'s in distances, angles and torsion angles; correlations between e.s.d.'s in cell parameters are only used when they are defined by crystal symmetry. An approximate (isotropic) treatment of cell e.s.d.'s is used for estimating e.s.d.'s involving 1.s. planes.

Refinement. Refinement of $F^{2}$ against ALL reflections. The weighted $R$-factor $w R$ and goodness of fit $S$ are based on $F^{2}$, conventional $R$-factors $R$ are based on $F$, with $F$ set to zero for negative $F^{2}$. The threshold expression of $F^{2}>\sigma\left(F^{2}\right)$ is used only for calculating $R$-factors(gt) $e t c$. and is not relevant to the choice of reflections for refinement. $R$-factors based on $F^{2}$ are statistically about twice as large as those based on $F$, and $R$ - factors based on ALL data will be even larger.

Fractional atomic coordinates and isotropic or equivalent isotropic displacement parameters $\left(\hat{A}^{2}\right)$

\begin{tabular}{|c|c|c|c|c|}
\hline & $x$ & $y$ & $z$ & $U_{\text {iso }} * / U_{\text {eq }}$ \\
\hline $\mathrm{O} 1$ & $0.24941(9)$ & $0.3021(3)$ & $0.56604(13)$ & $0.0306(5)$ \\
\hline $\mathrm{O} 2$ & $0.31408(9)$ & $0.3485(3)$ & $0.41718(12)$ & $0.0285(4)$ \\
\hline $\mathrm{O} 1 \mathrm{~W}$ & $0.45721(11)$ & $0.3587(4)$ & $0.36594(15)$ & $0.0360(5)$ \\
\hline $\mathrm{O} 2 \mathrm{~W}$ & $0.52898(12)$ & $-0.1307(4)$ & $0.37002(17)$ & $0.0398(5)$ \\
\hline N1 & $0.22255(10)$ & $0.8913(4)$ & $0.70511(15)$ & $0.0249(5)$ \\
\hline $\mathrm{H} 1 \mathrm{~N}$ & 0.2296 & 1.0021 & 0.6516 & $0.030 *$ \\
\hline $\mathrm{N} 2$ & $0.23986(10)$ & $0.5625(4)$ & $0.82389(15)$ & $0.0241(5)$ \\
\hline $\mathrm{H} 2 \mathrm{~N}$ & 0.2595 & 0.4266 & 0.8591 & $0.029 *$ \\
\hline $\mathrm{C} 1$ & $0.12302(14)$ & $0.6171(5)$ & 0.92071 (19) & $0.0290(6)$ \\
\hline H1A & 0.1303 & 0.4775 & 0.9723 & $0.035^{*}$ \\
\hline $\mathrm{C} 2$ & $0.06132(14)$ & $0.7751(5)$ & $0.9168(2)$ & $0.0331(7)$ \\
\hline $\mathrm{H} 2 \mathrm{~A}$ & 0.0264 & 0.7417 & 0.9673 & $0.040^{*}$ \\
\hline $\mathrm{C} 3$ & $0.04988(14)$ & $0.9848(5)$ & $0.8385(2)$ & $0.0334(7)$ \\
\hline $\mathrm{H} 3 \mathrm{~A}$ & 0.0074 & 1.0858 & 0.8380 & $0.040^{*}$ \\
\hline $\mathrm{C} 4$ & $0.09993(13)$ & $1.0447(5)$ & $0.7624(2)$ & $0.0300(6)$ \\
\hline $\mathrm{H} 4 \mathrm{~A}$ & 0.0924 & 1.1838 & 0.7107 & $0.036^{*}$ \\
\hline $\mathrm{C} 5$ & $0.16226(13)$ & 0.8870 & $0.76689(18)$ & $0.0237(6)$ \\
\hline C6 & $0.17353(13)$ & $0.6765(5)$ & 0.84417 (18) & $0.0238(6)$ \\
\hline $\mathrm{C} 7$ & $0.26826(13)$ & $0.6960(5)$ & $0.74162(18)$ & $0.0234(6)$ \\
\hline $\mathrm{C} 8$ & $0.34047(13)$ & $0.6448(5)$ & $0.69974(18)$ & $0.0264(6)$ \\
\hline H8A & 0.3582 & 0.4724 & 0.7285 & $0.032 *$ \\
\hline H8B & 0.3741 & 0.7816 & 0.7299 & $0.032 *$ \\
\hline C9 & $0.34097(13)$ & $0.6433(5)$ & $0.57180(18)$ & $0.0252(6)$ \\
\hline H9A & 0.3207 & 0.8115 & 0.5425 & $0.030 *$ \\
\hline
\end{tabular}


supporting information

\begin{tabular}{lllll} 
H9B & 0.3909 & 0.6334 & 0.5521 & $0.030^{*}$ \\
C10 & $0.29840(13)$ & $0.4120(5)$ & $0.51556(18)$ & $0.0227(6)$ \\
H1W1 & $0.4133(12)$ & $0.352(7)$ & $0.386(3)$ & $0.084(13)^{*}$ \\
H1W2 & $0.4755(16)$ & $0.208(4)$ & $0.384(3)$ & $0.059(11)^{*}$ \\
H2W1 & $0.5341(16)$ & $-0.149(6)$ & $0.3009(16)$ & $0.053(9)^{*}$ \\
H2W2 & $0.5073(19)$ & $-0.268(5)$ & $0.387(3)$ & $0.089(14)^{*}$ \\
\hline
\end{tabular}

Atomic displacement parameters $\left(\AA^{2}\right)$

\begin{tabular}{lllllll}
\hline & $U^{11}$ & $U^{22}$ & $U^{33}$ & $U^{12}$ & $U^{13}$ & $U^{23}$ \\
\hline O1 & $0.0340(10)$ & $0.0248(10)$ & $0.0334(9)$ & $-0.0060(8)$ & $0.0054(8)$ & $0.0027(8)$ \\
O2 & $0.0334(10)$ & $0.0268(10)$ & $0.0255(8)$ & $-0.0038(8)$ & $0.0042(8)$ & $-0.0056(7)$ \\
O1W & $0.0374(13)$ & $0.0303(12)$ & $0.0416(11)$ & $-0.0013(10)$ & $0.0106(10)$ & $-0.0001(9)$ \\
O2W & $0.0486(13)$ & $0.0315(12)$ & $0.0399(11)$ & $-0.0038(11)$ & $0.0079(10)$ & $-0.0010(10)$ \\
N1 & $0.0311(12)$ & $0.0209(12)$ & $0.0230(9)$ & $-0.0003(10)$ & $0.0032(9)$ & $0.0034(9)$ \\
N2 & $0.0290(12)$ & $0.0205(12)$ & $0.0225(9)$ & $0.0022(10)$ & $0.0013(9)$ & $0.0013(8)$ \\
C1 & $0.0336(15)$ & $0.0257(15)$ & $0.0281(12)$ & $-0.0059(13)$ & $0.0043(11)$ & $-0.0011(11)$ \\
C2 & $0.0315(15)$ & $0.0340(16)$ & $0.0348(14)$ & $-0.0053(13)$ & $0.0090(12)$ & $-0.0058(12)$ \\
C3 & $0.0292(15)$ & $0.0272(15)$ & $0.0438(15)$ & $0.0027(13)$ & $0.0035(13)$ & $-0.0062(13)$ \\
C4 & $0.0309(15)$ & $0.0231(15)$ & $0.0354(13)$ & $0.0009(12)$ & $-0.0006(12)$ & $-0.0010(11)$ \\
C5 & $0.0267(14)$ & $0.0194(14)$ & $0.0248(11)$ & $-0.0044(11)$ & $0.0016(11)$ & $-0.0032(10)$ \\
C6 & $0.0268(14)$ & $0.0198(13)$ & $0.0248(12)$ & $-0.0005(11)$ & $0.0010(11)$ & $-0.0029(10)$ \\
C7 & $0.0289(14)$ & $0.0202(13)$ & $0.0206(11)$ & $-0.0035(12)$ & $-0.0004(10)$ & $-0.0045(10)$ \\
C8 & $0.0240(14)$ & $0.0271(15)$ & $0.0279(12)$ & $-0.0009(12)$ & $0.0006(11)$ & $-0.0011(11)$ \\
C9 & $0.0289(14)$ & $0.0208(14)$ & $0.0264(12)$ & $-0.0028(12)$ & $0.0049(11)$ & $0.0005(11)$ \\
C10 & $0.0257(14)$ & $0.0167(13)$ & $0.0256(12)$ & $0.0048(11)$ & $0.0012(11)$ & $0.0050(10)$ \\
& & & & & & \\
\hline
\end{tabular}

Geometric parameters ( $\left.\AA,{ }^{\circ}\right)$

\begin{tabular}{llll}
\hline $\mathrm{O} 1-\mathrm{C} 10$ & $1.250(3)$ & $\mathrm{C} 2-\mathrm{C} 3$ & $1.404(4)$ \\
$\mathrm{O} 2-\mathrm{C} 10$ & $1.269(3)$ & $\mathrm{C} 2-\mathrm{H} 2 \mathrm{~A}$ & 0.9300 \\
$\mathrm{O} 1 \mathrm{~W}-\mathrm{H} 1 \mathrm{~W} 1$ & $0.863(18)$ & $\mathrm{C} 3-\mathrm{C} 4$ & $1.376(3)$ \\
$\mathrm{O} 1 \mathrm{~W}-\mathrm{H} 1 \mathrm{~W} 2$ & $0.845(18)$ & $\mathrm{C} 3-\mathrm{H} 3 \mathrm{~A}$ & 0.9300 \\
$\mathrm{O} 2 \mathrm{~W}-\mathrm{H} 2 \mathrm{~W} 1$ & $0.841(17)$ & $\mathrm{C} 4-\mathrm{C} 5$ & $1.389(3)$ \\
$\mathrm{O} 2 \mathrm{~W}-\mathrm{H} 2 \mathrm{~W} 2$ & $0.823(18)$ & $\mathrm{C} 4-\mathrm{H} 4 \mathrm{~A}$ & 0.9300 \\
$\mathrm{~N} 1-\mathrm{C} 7$ & $1.336(3)$ & $\mathrm{C} 5-\mathrm{C} 6$ & $1.399(3)$ \\
$\mathrm{N} 1-\mathrm{C} 5$ & $1.381(3)$ & $\mathrm{C} 7-\mathrm{C} 8$ & $1.481(3)$ \\
$\mathrm{N} 1-\mathrm{H} 1 \mathrm{~N}$ & 0.8600 & $\mathrm{C} 8-\mathrm{C} 9$ & $1.525(3)$ \\
$\mathrm{N} 2-\mathrm{C} 7$ & $1.325(3)$ & $\mathrm{C} 8-\mathrm{H} 8 \mathrm{~A}$ & 0.9700 \\
$\mathrm{~N} 2-\mathrm{C} 6$ & $1.387(3)$ & $\mathrm{C} 8-\mathrm{H} 8 \mathrm{~B}$ & 0.9700 \\
$\mathrm{~N} 2-\mathrm{H} 2 \mathrm{~N}$ & 0.8600 & $\mathrm{C} 9-\mathrm{C} 10$ & $1.518(3)$ \\
$\mathrm{C} 1-\mathrm{C} 2$ & $1.380(3)$ & $\mathrm{C} 9-\mathrm{H} 9 \mathrm{~A}$ & 0.9700 \\
$\mathrm{C} 1-\mathrm{C} 6$ & $1.386(3)$ & $\mathrm{C} 9-\mathrm{H} 9 \mathrm{~B}$ & 0.9700 \\
$\mathrm{C} 1-\mathrm{H} 1 \mathrm{~A}$ & 0.9300 & & $121.8(2)$ \\
& & & $132.5(2)$ \\
$\mathrm{H} 1 \mathrm{~W} 1-\mathrm{O} 1 \mathrm{~W}-\mathrm{H} 1 \mathrm{~W} 2$ & $105(3)$ & $\mathrm{C} 4-\mathrm{C} 5-\mathrm{C} 6$ & $121.3(2)$ \\
$\mathrm{H} 2 \mathrm{~W} 1-\mathrm{O} 2 \mathrm{~W}-\mathrm{H} 2 \mathrm{~W} 2$ & $104(3)$ & $\mathrm{C} 1-\mathrm{C} 6-\mathrm{C} 5$ & \\
$\mathrm{C} 7-\mathrm{N} 1-\mathrm{C} 5$ & $109.22(19)$ & &
\end{tabular}




\begin{tabular}{|c|c|c|c|}
\hline $\mathrm{C} 7-\mathrm{N} 1-\mathrm{H} 1 \mathrm{~N}$ & 125.4 & $\mathrm{~N} 2-\mathrm{C} 6-\mathrm{C} 5$ & $106.2(2)$ \\
\hline $\mathrm{C} 5-\mathrm{N} 1-\mathrm{H} 1 \mathrm{~N}$ & 125.4 & $\mathrm{~N} 2-\mathrm{C} 7-\mathrm{N} 1$ & $109.2(2)$ \\
\hline $\mathrm{C} 7-\mathrm{N} 2-\mathrm{C} 6$ & $109.2(2)$ & $\mathrm{N} 2-\mathrm{C} 7-\mathrm{C} 8$ & $125.6(2)$ \\
\hline $\mathrm{C} 7-\mathrm{N} 2-\mathrm{H} 2 \mathrm{~N}$ & 125.4 & $\mathrm{~N} 1-\mathrm{C} 7-\mathrm{C} 8$ & $125.2(2)$ \\
\hline $\mathrm{C} 6-\mathrm{N} 2-\mathrm{H} 2 \mathrm{~N}$ & 125.4 & $\mathrm{C} 7-\mathrm{C} 8-\mathrm{C} 9$ & $114.5(2)$ \\
\hline $\mathrm{C} 2-\mathrm{C} 1-\mathrm{C} 6$ & $116.9(2)$ & $\mathrm{C} 7-\mathrm{C} 8-\mathrm{H} 8 \mathrm{~A}$ & 108.6 \\
\hline $\mathrm{C} 2-\mathrm{C} 1-\mathrm{H} 1 \mathrm{~A}$ & 121.6 & $\mathrm{C} 9-\mathrm{C} 8-\mathrm{H} 8 \mathrm{~A}$ & 108.6 \\
\hline $\mathrm{C} 6-\mathrm{C} 1-\mathrm{H} 1 \mathrm{~A}$ & 121.6 & $\mathrm{C} 7-\mathrm{C} 8-\mathrm{H} 8 \mathrm{~B}$ & 108.6 \\
\hline $\mathrm{C} 1-\mathrm{C} 2-\mathrm{C} 3$ & $121.7(2)$ & $\mathrm{C} 9-\mathrm{C} 8-\mathrm{H} 8 \mathrm{~B}$ & 108.6 \\
\hline $\mathrm{C} 1-\mathrm{C} 2-\mathrm{H} 2 \mathrm{~A}$ & 119.2 & $\mathrm{H} 8 \mathrm{~A}-\mathrm{C} 8-\mathrm{H} 8 \mathrm{~B}$ & 107.6 \\
\hline $\mathrm{C} 3-\mathrm{C} 2-\mathrm{H} 2 \mathrm{~A}$ & 119.2 & $\mathrm{C} 10-\mathrm{C} 9-\mathrm{C} 8$ & $113.63(19)$ \\
\hline $\mathrm{C} 4-\mathrm{C} 3-\mathrm{C} 2$ & $121.6(2)$ & $\mathrm{C} 10-\mathrm{C} 9-\mathrm{H} 9 \mathrm{~A}$ & 108.8 \\
\hline $\mathrm{C} 4-\mathrm{C} 3-\mathrm{H} 3 \mathrm{~A}$ & 119.2 & $\mathrm{C} 8-\mathrm{C} 9-\mathrm{H} 9 \mathrm{~A}$ & 108.8 \\
\hline $\mathrm{C} 2-\mathrm{C} 3-\mathrm{H} 3 \mathrm{~A}$ & 119.2 & $\mathrm{C} 10-\mathrm{C} 9-\mathrm{H} 9 \mathrm{~B}$ & 108.8 \\
\hline $\mathrm{C} 3-\mathrm{C} 4-\mathrm{C} 5$ & $116.7(2)$ & $\mathrm{C} 8-\mathrm{C} 9-\mathrm{H} 9 \mathrm{~B}$ & 108.8 \\
\hline $\mathrm{C} 3-\mathrm{C} 4-\mathrm{H} 4 \mathrm{~A}$ & 121.7 & $\mathrm{H} 9 \mathrm{~A}-\mathrm{C} 9-\mathrm{H} 9 \mathrm{~B}$ & 107.7 \\
\hline $\mathrm{C} 5-\mathrm{C} 4-\mathrm{H} 4 \mathrm{~A}$ & 121.7 & $\mathrm{O} 1-\mathrm{C} 10-\mathrm{O} 2$ & $124.2(2)$ \\
\hline $\mathrm{N} 1-\mathrm{C} 5-\mathrm{C} 4$ & $132.0(2)$ & $\mathrm{O} 1-\mathrm{C} 10-\mathrm{C} 9$ & $119.2(2)$ \\
\hline $\mathrm{N} 1-\mathrm{C} 5-\mathrm{C} 6$ & $106.1(2)$ & $\mathrm{O} 2-\mathrm{C} 10-\mathrm{C} 9$ & $116.6(2)$ \\
\hline $\mathrm{C} 6-\mathrm{C} 1-\mathrm{C} 2-\mathrm{C} 3$ & $0.5(4)$ & $\mathrm{C} 4-\mathrm{C} 5-\mathrm{C} 6-\mathrm{C} 1$ & $-0.5(3)$ \\
\hline $\mathrm{C} 1-\mathrm{C} 2-\mathrm{C} 3-\mathrm{C} 4$ & $-0.6(4)$ & $\mathrm{N} 1-\mathrm{C} 5-\mathrm{C} 6-\mathrm{N} 2$ & $-0.7(2)$ \\
\hline $\mathrm{C} 2-\mathrm{C} 3-\mathrm{C} 4-\mathrm{C} 5$ & $0.1(4)$ & $\mathrm{C} 4-\mathrm{C} 5-\mathrm{C} 6-\mathrm{N} 2$ & $179.2(2)$ \\
\hline $\mathrm{C} 7-\mathrm{N} 1-\mathrm{C} 5-\mathrm{C} 4$ & $-179.9(2)$ & $\mathrm{C} 6-\mathrm{N} 2-\mathrm{C} 7-\mathrm{N} 1$ & $-1.2(3)$ \\
\hline $\mathrm{C} 7-\mathrm{N} 1-\mathrm{C} 5-\mathrm{C} 6$ & $0.0(2)$ & $\mathrm{C} 6-\mathrm{N} 2-\mathrm{C} 7-\mathrm{C} 8$ & $176.5(2)$ \\
\hline $\mathrm{C} 3-\mathrm{C} 4-\mathrm{C} 5-\mathrm{N} 1$ & $-179.6(2)$ & $\mathrm{C} 5-\mathrm{N} 1-\mathrm{C} 7-\mathrm{N} 2$ & $0.7(2)$ \\
\hline $\mathrm{C} 3-\mathrm{C} 4-\mathrm{C} 5-\mathrm{C} 6$ & $0.4(3)$ & $\mathrm{C} 5-\mathrm{N} 1-\mathrm{C} 7-\mathrm{C} 8$ & $-177.0(2)$ \\
\hline $\mathrm{C} 2-\mathrm{C} 1-\mathrm{C} 6-\mathrm{N} 2$ & $-179.6(2)$ & $\mathrm{N} 2-\mathrm{C} 7-\mathrm{C} 8-\mathrm{C} 9$ & $135.7(2)$ \\
\hline $\mathrm{C} 2-\mathrm{C} 1-\mathrm{C} 6-\mathrm{C} 5$ & $0.1(3)$ & $\mathrm{N} 1-\mathrm{C} 7-\mathrm{C} 8-\mathrm{C} 9$ & $-47.0(3)$ \\
\hline $\mathrm{C} 7-\mathrm{N} 2-\mathrm{C} 6-\mathrm{C} 1$ & $-179.1(2)$ & $\mathrm{C} 7-\mathrm{C} 8-\mathrm{C} 9-\mathrm{C} 10$ & $-66.3(3)$ \\
\hline $\mathrm{C} 7-\mathrm{N} 2-\mathrm{C} 6-\mathrm{C} 5$ & $1.2(2)$ & $\mathrm{C} 8-\mathrm{C} 9-\mathrm{C} 10-\mathrm{O} 1$ & $23.1(3)$ \\
\hline $\mathrm{N} 1-\mathrm{C} 5-\mathrm{C} 6-\mathrm{C} 1$ & $179.5(2)$ & $\mathrm{C} 8-\mathrm{C} 9-\mathrm{C} 10-\mathrm{O} 2$ & $-159.0(2)$ \\
\hline
\end{tabular}

Hydrogen-bond geometry $\left(\AA,{ }^{\circ}\right)$

\begin{tabular}{lllll}
\hline$D-\mathrm{H} \cdots A$ & $D-\mathrm{H}$ & $\mathrm{H} \cdots A$ & $D \cdots A$ & $D-\mathrm{H} \cdots A$ \\
\hline $\mathrm{N} 1-\mathrm{H} 1 N \cdots \mathrm{O} 1^{\mathrm{i}}$ & 0.86 & 1.86 & $2.700(2)$ & 166 \\
$\mathrm{~N} 2-\mathrm{H} 2 N \cdots \mathrm{O} 2^{\mathrm{ii}}$ & 0.86 & 1.80 & $2.654(3)$ & 170 \\
$\mathrm{O} 2 W-\mathrm{H} 2 W 1^{\cdots} \mathrm{O} 1 W^{\mathrm{iii}}$ & $0.84(2)$ & $2.01(2)$ & $2.842(3)$ & $172(3)$ \\
\hline
\end{tabular}

Symmetry codes: (i) $x, y+1, z$; (ii) $x,-y+1 / 2, z+1 / 2$; (iii) $-x+1, y-1 / 2,-z+1 / 2$. 